\title{
Norois
}

Environnement, aménagement, société

$191 \mid 2004 / 2$

Les types de temps

\section{Recherche sur les types de temps associes aux brises de mer}

Une méthode d'analyse par téledetection

Research on weather types related to sea breezes: a method of analysis using remote sensing

Olivier Planchon, Vincent Dubreuil, Frédéric Damato, Pascal Gouéry et Sébastien Decaux

\section{OpenEdition}

\section{Journals}

\section{Édition électronique}

URL : http://journals.openedition.org/norois/1027

DOI : 10.4000/norois. 1027

ISBN : 978-2-7535-1539-0

ISSN : $1760-8546$

Éditeur

Presses universitaires de Rennes

Édition imprimée

Date de publication : 1 mars 2004

Pagination : $59-74$

ISBN : $978-2-86847-977-8$

ISSN : 0029-182X

Référence électronique

Olivier Planchon, Vincent Dubreuil, Frédéric Damato, Pascal Gouéry et Sébastien Decaux, «Recherche sur les types de temps associes aux brises de mer », Norois [En ligne], 191 | 2004/2, mis en ligne le 01 septembre 2008, consulté le 19 avril 2019. URL : http://journals.openedition.org/norois/1027 ; DOI : $10.4000 /$ norois. 1027

Ce document a été généré automatiquement le 19 avril 2019

(c) Tous droits réservés 


\title{
Recherche sur les types de temps associes aux brises de mer
}

\author{
Une méthode d'analyse par téledetection \\ Research on weather types related to sea breezes: a method of analysis using \\ remote sensing
}

\author{
Olivier Planchon, Vincent Dubreuil, Frédéric Damato, Pascal Gouéry et \\ Sébastien Decaux
}

\section{Introduction}

1 Les circulations de brise de mer favorisent l'apparition de certains types de temps propres au littoral et à une bordure continentale d'extension variable. Ces types de temps particuliers, relativement frais, humides mais le plus souvent ensoleillés par rapport aux régions intérieures, ont de nombreuses répercussions sur les activités humaines. Certaines sont déjà bien connues (tourisme balnéaire), très étudiées (pollution atmosphérique, accrue par la stabilité de l'air maritime : très nombreuses études citées par exemple par Abbs \& Physick 1992; Simpson 1994; Janoueix-Yacono 1995), parfois plus inattendues (effets bénéfiques sur la maturation du raisin, en Afrique du Sud par exemple: Planchon et al., 2000; Bonnardot et al., 2002). Diverses méthodes ont été appliquées à l'étude des circulations de brises de mer, à leurs effets sur les conditions météorologiques aux échelles régionales et locales (observation météorologique, analyse statistique, modélisation numérique méso-échelle: Simpson, 1994). La télédétection permet de mettre en évidence certaines manifestations nuageuses caractéristiques de la brise de mer (front de brise), mais a le plus souvent été utilisée comme illustration plus que comme moyen de calcul et d'analyse. Une méthode d'identification des fronts de brise par télédétection a ainsi été développée et appliquée à l'Europe de l'Ouest et au nord-est du Brésil, afin d'en calculer la fréquence d'apparition et la distance préférentielle de pénétration dans les terres aux heures chaudes de la journée. 


\section{Brise de mer et types de temps : rappels et quelques particularités dans l'ouest de la France}

\section{Mécanismes et observations}

2 L'échauffement différentiel des océans et des continents induit des contrastes thermiques plus ou moins importants entre les masses d'air qui les surmontent, ce qui permet le développement des circulations de brise de mer (à condition que le vent synoptique y soit favorable). Les conditions favorables au développement de la brise de mer sont surtout liées à l'échauffement diurne rapide et plus ou moins intense du continent, alors que la température de surface de la mer dans le même temps est pratiquement constante. Au lever du jour, dès qu'un gradient thermique d'environ 1 à 3 degrés est observé entre la surface du continent et celle de la mer, des circulations de brise d'échelle locale se mettent en place (Borne et al., 1998 ; Mayençon, 1992). La pénétration de ces brises dans les terres est limitée à quelques kilomètres au maximum. Ce phénomène est donc très directement et étroitement associé au littoral et à la discontinuité terre-mer. Le développement d'une circulation de brise de mer proprement dite, d'échelle régionale et capable de pénétrer à plusieurs dizaines de kilomètres dans les terres, nécessite un gradient thermique d'au moins $4^{\circ} \mathrm{C}$ aux latitudes intertropicales (Okoola, 1978 ; Cautenet et Rosset, 1989) et $6^{\circ} \mathrm{C}$ aux hautes et moyennes latitudes (Cautenet, 1988). Ce gradient thermique est donc atteint plus tard dans la journée.

Figure 1 : Schéma de la circulation de brise de mer

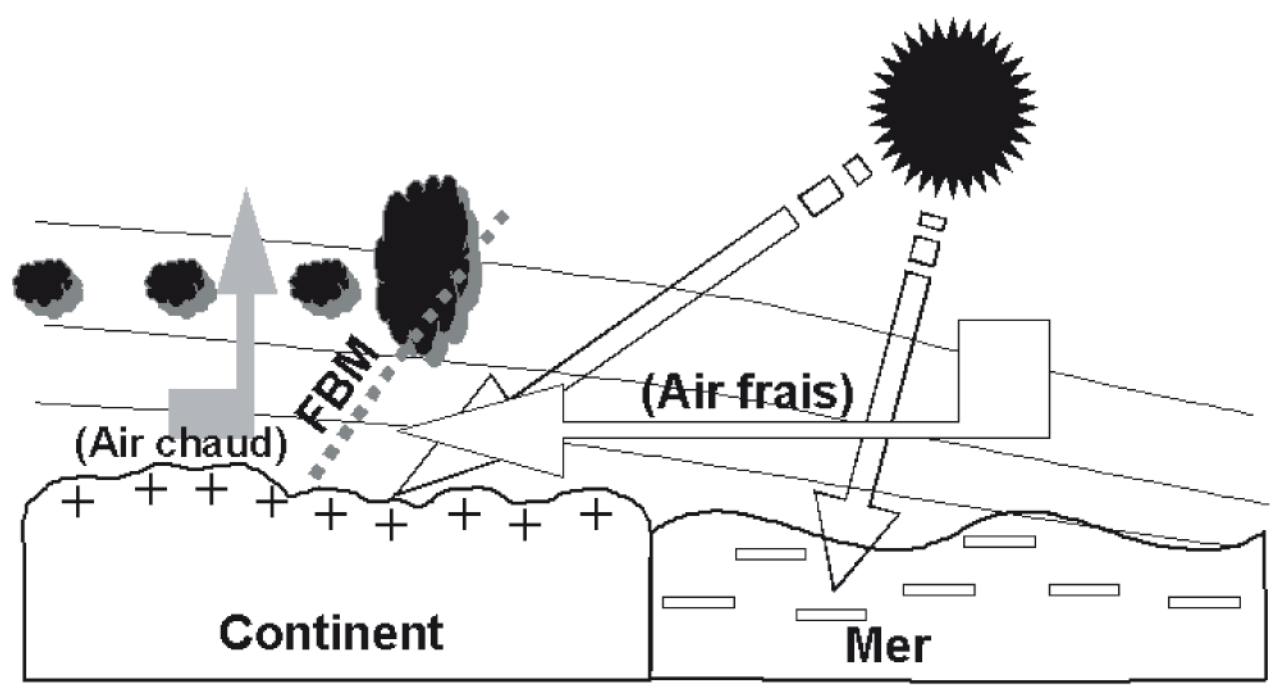

3 L'air au-dessus de la terre s'échauffe vite pendant la journée, s'élève, et la pression diminue en surface, par rapport à celle observée sur mer au même niveau. Avec le gradient de pression horizontal ainsi créé, un mouvement d'air plus frais et plus dense, dirigé de la mer vers la terre, s'organise près de la surface : la brise de mer. Aux latitudes intertropicales, l'intensité de l'échauffement diurne continental et la moindre influence de la force de Coriolis favorisent, quand le relief le permet, la pénétration de la brise dans l'intérieur des terres jusqu'à 100 ou $150 \mathrm{~km}$ alors qu'aux latitudes extra-tropicales, la pénétration de la brise excède rarement 40 à $50 \mathrm{~km}$ (Yan et Anthes, 1987; Abbs et 
Physick, 1992). Dans les zones tempérées, la brise de mer est une circulation de saison chaude tandis qu'elle peut se manifester toute l'année en zone intertropicale. Dans les climats tropicaux à alternance saisonnière saison des pluies/saison sèche, la saison sèche (ou peu pluvieuse) est la plus favorable au développement de circulations de brise de mer puissantes, compte-tenu de la plus forte insolation. En pénétrant dans les terres, la brise de mer intervient largement sur les éléments du climat (température, humidité, nébulosité, précipitation). L'arrivée de la brise de mer sur le littoral se traduit habituellement par un changement de la direction du vent en surface (d'une simple déviation à un renversement complet, en fonction de la direction du vent dominant) associée à un renforcement de sa vitesse, une baisse de la température, une augmentation de l'humidité relative et un changement de l'état du ciel : l'air maritime frais et stable impose habituellement un ciel dégagé au-dessus de l'espace touché par la brise, ou apporte éventuellement brumes, brouillards ou nuages bas stratiformes, en particulier dans le cas de bordures continentales baignées par des eaux de surface froides (upwellings côtiers, par exemple). Les types de temps associés aux brises de mer sont variés aux latitudes extra-tropicales, car ils sont influencés par la circulation d'échelle synoptique aux multiples configurations, même en saison chaude. Ainsi, toutes les caractéristiques définies ci-dessus ne sont pas forcément réunies sur une station donnée.

Figure 2 : Trois journées de brise de mer à Dinard. Manifestations et effets de la brise de mer en surface sur la température, l'humidité relative et le vent (vitesse et direction).

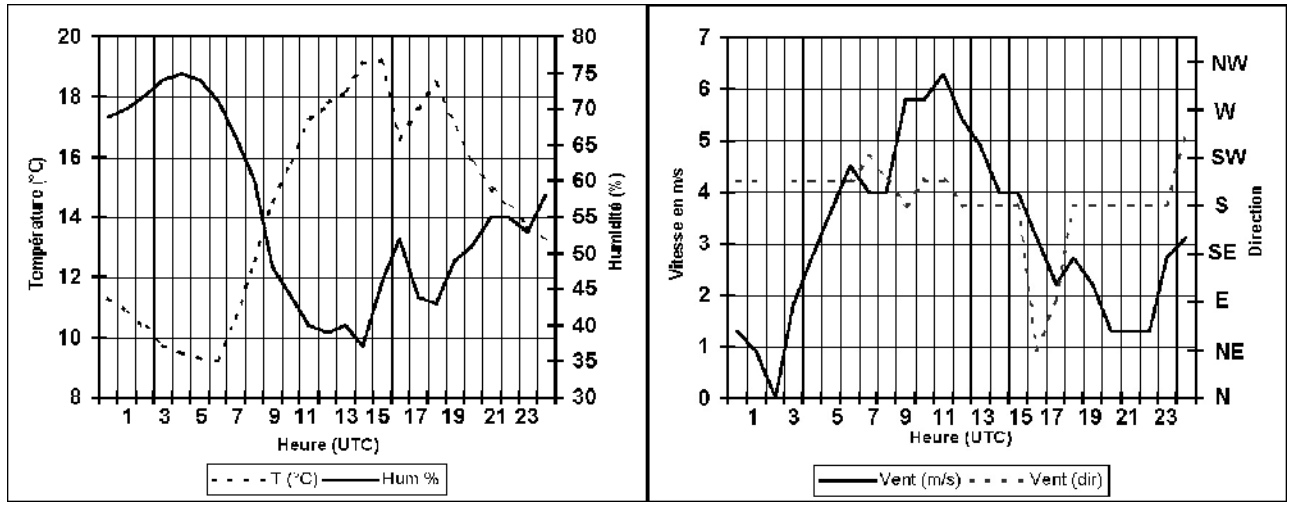

a) Journée du 21 avril 2002

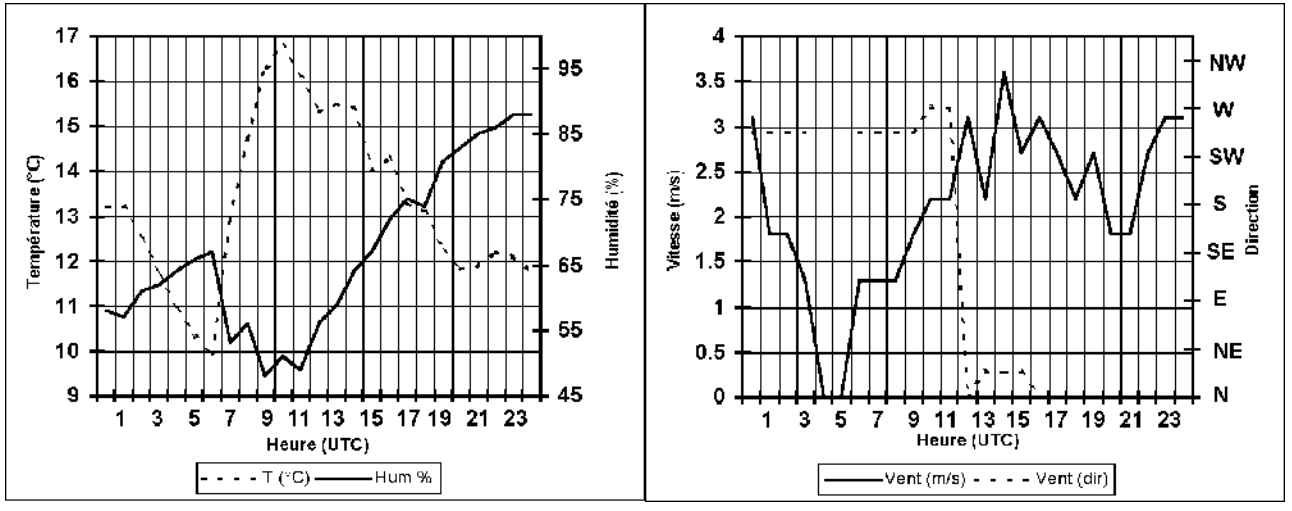

b) Journée du 22 avril 2002 


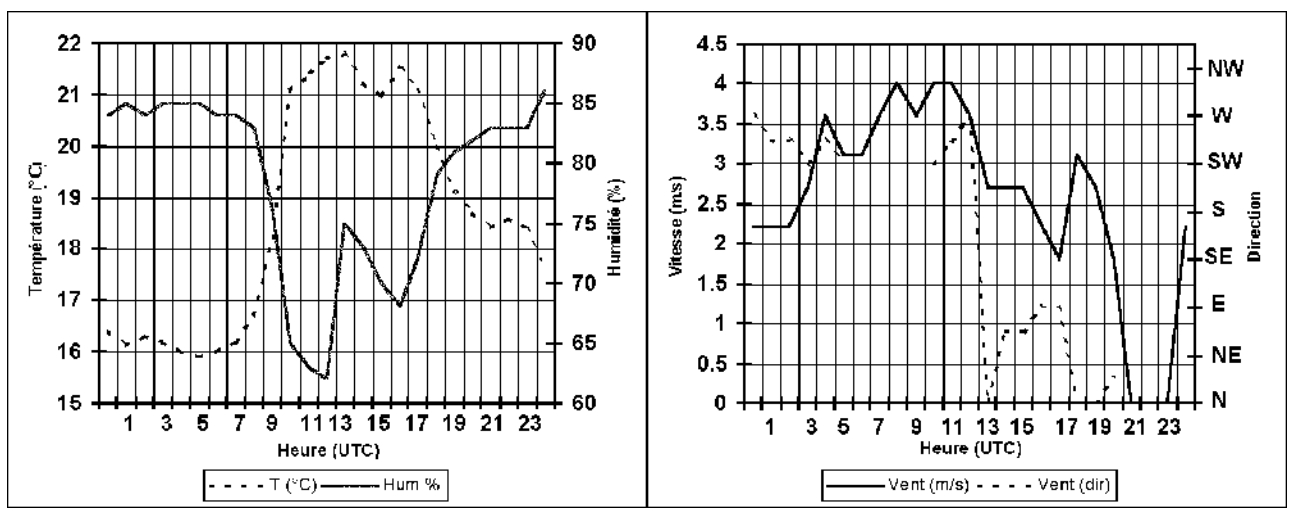

c) Journée du 30 août 2002

4 A Dinard, sur la côte nord de la Bretagne (golfe normand-breton), les données horaires du 21 avril 2002 (figure 2a) montraient bien, dans l'après-midi, une baisse caractéristique de la température associée à une augmentation de l'humidité relative, ne pouvant être dues à une averse puisque le ciel était dégagé ce jour-là. En revanche, au renversement, caractéristique également mais de courte durée, de la direction du vent de sud à nord-est (au printemps et en été, à la station de Pleurtuit, à $6 \mathrm{~km}$ de la côte, le vent s'oriente habituellement du sud-ouest à 09:00 UTC au secteur nord à 15:00 UTC : Decaux, 2002), ne correspondait pas un renforcement de sa vitesse : c'est une diminution qui était observée. Le 22 avril (figure 2 b), alors qu'un renforcement de la vitesse du vent accompagnait, vers midi, le changement de direction, de l'ouest au nord, la baisse de température n'a pas été accompagnée d'une augmentation simultanée et significative de l'humidité relative. Le 30 août (figure 2c), le rythme diurne de la température, de l'humidité et du vent rappelait les observations du 21 avril, un affaiblissement de la vitesse du vent accompagnant son changement de direction (ouest à nord) entre midi et 13:00 UTC (ouest à nord).

5 A l'avant de la circulation, le front de brise de mer est une discontinuité spatiale et atmosphérique méso-échelle marquant un contraste thermodynamique. L'air marin plus dense, stable et frais soulève l'air continental plus chaud et instable, ce qui active la convection au niveau du front de brise et permet la formation d'une ligne de nuages de type cumuliforme, parallèle à la côte et poussée vers l'intérieur par la brise de mer (figure 1). Grâce à cette nébulosité caractéristique sur l'intérieur des terres, le front de brise de mer est souvent détectable sur les images satellites, tandis que le littoral et la bordure du continent bénéficient d'un ciel dégagé. Afin d'estimer la fréquence d'apparition de ces types de temps liés aux circulations de brise de mer, l'évolution des fronts de brise a été suivie dans l'espace et dans le temps par télédétection, durant les saisons les plus favorables (saison chaude en Europe occidentale et saison la moins arrosée dans le NordEst du Brésil), de façon de mettre en évidence les variations mensuelles imposées par les états de surface et par les conditions climatiques d'échelle synoptique.

\section{Brise de mer et télédétection}

Dans plusieurs études, les fronts de brise de mer ont été identifiés par l'imagerie satellitaire, en repérant les formations nuageuses caractéristiques qui leur sont associées. R. Pielke (1974) a utilisé les images satellites afin d'appuyer une analyse de la convection nuageuse et des orages associés au front de brise de mer en Floride. En Afrique de l'Ouest (golfe de Guinée), S. Cautenet et R. Rosset (1989) ont utilisé les images visibles du satellite 
METEOSAT pour illustrer certaines observations de pénétration des fronts de brise de mer pendant la saison sèche. J.E. Simpson (1994) a confirmé l'utilité des images satellites (NOAA 6 et 11) pour identifier la nébulosité liée au front de brise de mer sur différentes péninsules et bordures continentales (Sud de l'Angleterre, Danemark). La pénétration de la brise de mer dans l'intérieur des terres modifie la distribution spatiale des nuages sur la bordure du continent. A partir de l'imagerie satellitaire, le front de brise de mer apparaît souvent comme une ligne de nuages cumuliformes à l'intérieur du continent, mais toutes les situations de brise ne sont pas accompagnées d'un front nuageux (par exemple lorsque l'humidité de l'air est trop faible), ou celui-ci peut être masqué par d'autres formations nuageuses, d'échelle plus large. L'utilisation de la télédétection est donc restrictive, car seules les situations de brise de mer associées à un front nuageux peuvent être observées. Le suivi par satellite aide cependant à localiser la position de cette discontinuité et sa morphologie, c'est-à-dire indirectement à mesurer l'intensité et le caractère persistant de la brise.

Figure 3 : Image visible NOAA-12 du 30 août 2000 à 16:04 UTC

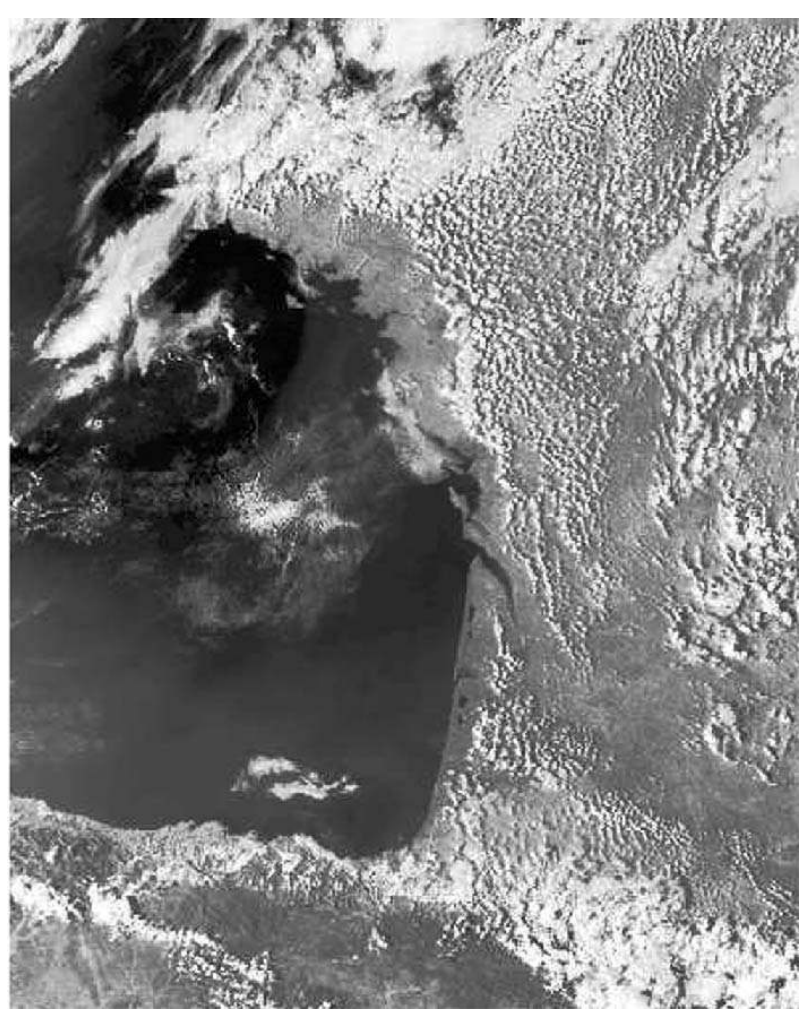

7 L'image du 30 août 2000 dans l'après-midi montrait un ciel dégagé au-dessus de la mer et parsemé de nuages cumuliformes au-dessus du continent, à l'intérieur des terres. La transition entre les cumulus et le ciel dégagé aide à localiser et à tracer le front de brise de mer, donc à mesurer la distance de pénétration de la brise de mer dans l'intérieur des terres. Un front de brise quasi continu apparaissait ainsi le long de la côte atlantique française, à une distance (40 km environ) qui correspond à la pénétration maximale habituellement observée aux latitudes moyennes. La ligne de cumulus mieux marquée dans certains secteurs (Charente Maritime, Aquitaine) souligne la position du front de brise et délimite ainsi nettement l'avancée de l'air maritime sur la bordure du continent. La température maximale atteignait $27^{\circ} \mathrm{C}$ à Bordeaux, $23^{\circ} \mathrm{C}$ à Nantes et $26^{\circ} \mathrm{C}$ à Rennes. 
8 La brise de mer ne garantit pas toujours un temps à ciel dégagé sur le littoral et la bordure touchée par l'air maritime. Au printemps et au début de l'été, sur les espaces maritimes d'Europe du nord-ouest, certaines situations météorologiques particulières favorisent la formation de vastes nappes de stratus bas sur la mer dont la surface est encore fraîche. Le phénomène est connu sous le nom de haar à l'est de la Grande-Bretagne (Smith, 1976 ; O'Hare et Sweeney, 1994). Avec des hautes pressions de surface sur l'Europe du Nord, des vents d'est dirigent vers la Grande Bretagne une masse d'air maritime stable rafraîchie au-dessus de la mer du Nord. La brise de mer, puissante en cette saison en Europe du nord-ouest (insolation élévée et gradient thermique terre-mer le plus accentué) peut permettre à ces brouillards d'advection ou nappes de stratus bas de s'avancer vers l'intérieur, où ils se dissipent avec le réchauffement diurne dans la journée, alors que le haar persiste sur la côte. Ce type de temps existe aussi sur la Manche, par exemple le 24 avril 2002 (figure 4).

Figure 4 : extension d'un stratus bas favorisée par la brise de mer

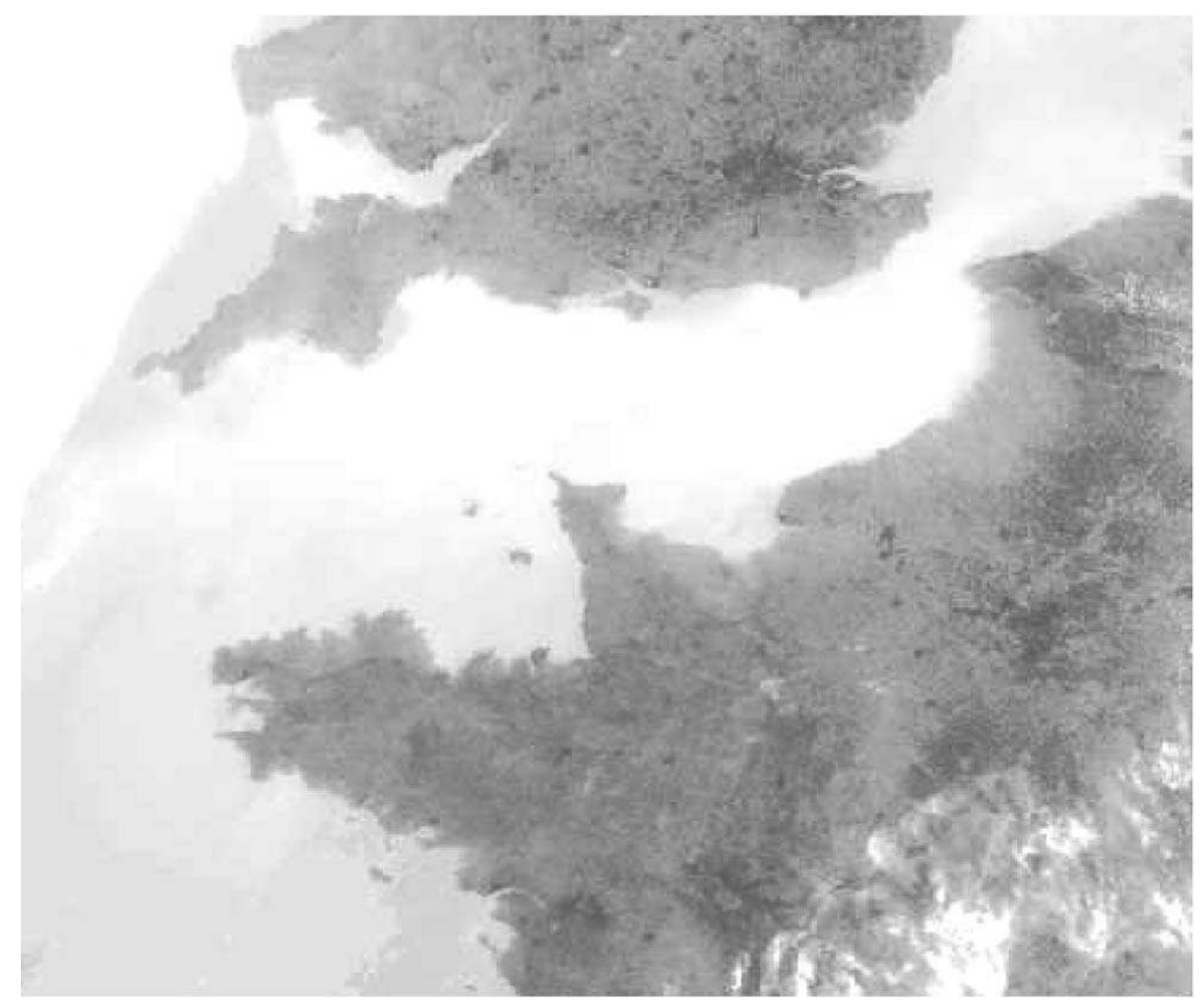

a) Image IRT NOAA-12, 24 avril 2002 à 15:36 UTC 


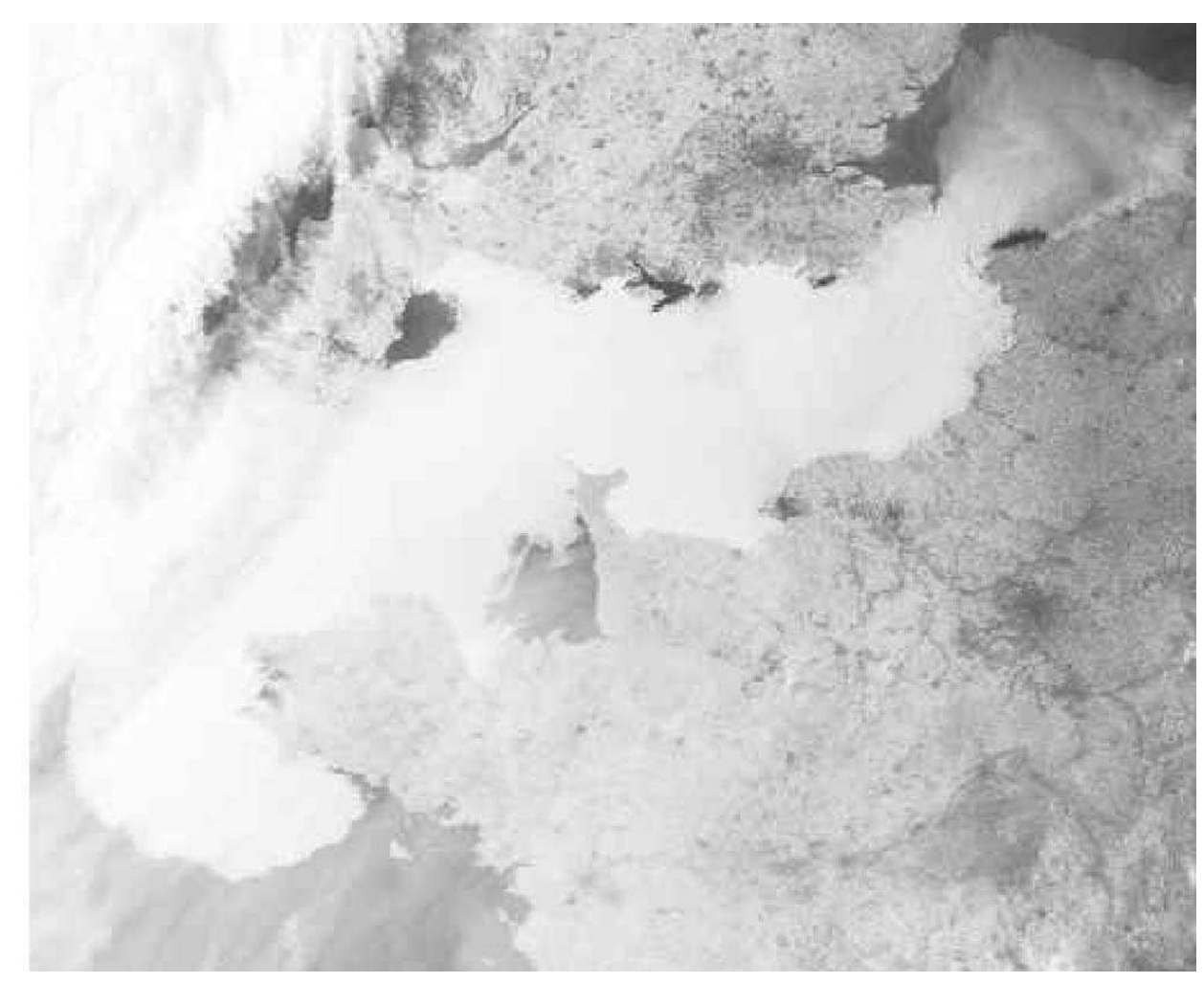

b) Image VIS NOAA-12, 24 avril 2002 à 17:49 UTC

Dans la journée du 24 avril 2002 (hautes pressions de surface sur l'ensemble de l'Europe occidentale), la pénétration des stratus présents sur la Manche était favorisée en fin d'après-midi par la généralisation des circulations de brise de mer, sur la bordure française surtout, mais aussi sur quelques régions anglaises (Devon, Dorset et Sussex). L'extention de la nappe de stratus sur des bordures continentales diversement exposées, ou même opposées, prouve le rôle joué par les circulations de brise de mer dans la généralisation d'un type de temps frais à ciel couvert autour de la Manche. L'absence de nuages bas sur le golfe normand-breton s'explique vraisemblablement par ses eaux de surface habituellement plus chaudes (Mounier et Gouéry, 1992). L'arrivée de la nappe de stratus sur les stations de la bordure du continent (intérieur immédiat) a provoqué une baisse d'autant plus sensible et brutale de la température que celle-ci était déjà relativement élevée en début d'après-midi, par exemple à Saint-Brieuc: la station de Trémuson (altitude $136 \mathrm{~m}$ ), située à $8 \mathrm{~km}$ de la côte (exposée au nord-est), a enregistré $22,1^{\circ} \mathrm{C}$ à $14: 00$ UTC par vent de sud-ouest, puis $13,8^{\circ} \mathrm{C}$ à 16:00 UTC par vent de nord-est. A $12 \mathrm{~km}$ de la côte et à l'est de Saint-Brieuc, la station de Quintenic qui échappait aux stratus enregistrait $21,1^{\circ} \mathrm{C}$ le même jour à 16:00 UTC (Decaux, 2002).

Ce type de temps à brouillards ou stratus bas maritimes est fréquent en été le long des côtes septentrionales de la Bretagne (Mounier, 1982), mais n'est pas le plus fréquemment associé aux circulations de brise de mer autour de la Manche. Au printemps et en été, les journées à ciel dégagé sur les espaces littoraux et à nuages cumuliformes sur l'intérieur sont plus représentatives des situations à brise de mer. Ce sont ces types de temps qui ont été analysés par télédétection. 


\section{Données et méthodes}

11 Les données utilisées pour l'étude des fronts de brise autour des côtes de la Manche ont été enregistrées par le satellite à défilement NOAA-14, placé sur une orbite quasi-polaire. Le satellite NOAA-14 dispose du Advanced Very High Resolution Radiometer (AVHRR), qui enregistre les détails de la surface à une résolution de $1.1 \mathrm{~km}$ à la verticale du satellite. Les images ont été acquises auprès de la SAA (Satellite Active Archive) de la NOAA (http:// www.saa.noaa.gov). Sept images seulement étaient manquantes durant la période d'étude. Les données traitées dans le cadre de l'étude sur le nord-est du Brésil ont été enregistrées par le satellite géostationnaire GOES-8, positionné à $75^{\circ} \mathrm{W}$ et donnant ainsi une vision de l'ensemble de l'Amérique du Sud. La résolution spatiale est de $1 \mathrm{~km}$ dans le canal visible et de $4 \mathrm{~km}$ dans l'infrarouge. Les fichiers ont été fournis par le SATMOS (CMS, Lannion, France). Les données infrarouge ont été utilisées pour estimer le gradient thermique mensuel moyen sur le nord-est du Brésil, entre le continent et l'océan selon deux transects perpendiculaires à la côte (Ceará et Pernambuco).

Les images visibles ont été privilégiées (longueurs d'onde comprises entre 0.58 et 0.68 $\mu \mathrm{m})$, car les enregistrements effectués dans ces longueurs d'onde permettent de discriminer les types de nuages en fonction de leur albedo : celui-ci varie notamment en fonction de l'épaisseur du nuage et de sa densité. Les images ainsi obtenues permettent d'identifier nettement la forme, l'extension et les contours des nuages et des formations nuageuses, et de bien distinguer les nuages bas des surfaces océaniques et continentales. Les images de l'Europe de l'ouest sélectionnées pour l'étude des fronts de brise autour de la manche étaient enregistrées entre 13:17 et 15:06 UTC, de mai à septembre 2000 inclus (mois de "saison chaude"). Pour le nord-est du Brésil, les données étaient enregistrées tous les jours à 18:00 UTC (15:00 locales dans le nord-est du Brésil) pour les mois de septembre à décembre 2000, c'est-à-dire les mois les moins arrosés sur une grande partie de l'espace étudié (Ratisbona, 1976 ; Nimer, 1989). La méthode utilisée consiste à identifier la nébulosité susceptible d'être liée aux fronts de brise (lignes de cumulus parallèles à la côte, sur le continent : figures $5 \mathrm{a}$ et $6 \mathrm{a}$ ), puis à tracer les fronts de brise observés sur des cartes afin d'en estimer la fréquence d'apparition.

Chaque image quotidienne a été géométriquement corrigée et projetée dans le système de référence «plate carrée» (latitude, longitude). Les fronts de brise de mer ont été identifiés et tracés sur chaque image; tous les fichiers-vecteur géoréférencés ont alors été superposés sur des cartes de l'Europe de l'ouest (figure 5b) et du nord-est du Brésil (figure 6b). La cartographie de la fréquence des fronts de brise a été réalisée en effectuant la somme des fichiers-vecteur. Afin d'effectuer l'analyse spatiale fondée sur les pixels, les fichiers-vecteur ont été convertis en fichiers-raster, en attribuant la valeur « 1 » aux pixels correspondant à une observation de front de brise de mer et « 0 » aux autres pixels. Un filtre moyen $3 \times 3$ a été appliqué pour lisser et homogénéiser les contours de l'image. Cette méthode de repérage des fronts de brise de mer par télédétection exclut d'une part les journées à situations perturbées, durant lesquelles les images sont en grande partie couvertes par les vastes formations nuageuses d'échelle synoptique, d'autre part les journées anticycloniques et très stables, sans nuages. Cette méthode a d'abord été appliquée à l'Europe de l'ouest entre 36 et $60^{\circ} \mathrm{N}$ et entre $10^{\circ} \mathrm{W}$ et $10^{\circ} \mathrm{E}$ (Damato et al., 2003a) puis au nord-est du Brésil, entre l'équateur et $15^{\circ} \mathrm{S}$ et entre 35 et $45^{\circ} \mathrm{W}$ (Damato et al., 2003b). Puis, une analyse a été effectuée de façon plus approfondie et à une échelle 
plus fine, autour de la Manche et du Pas de Calais, d'une part, et sur la bordure du Nordeste exposée au nord.

Figure 5 : Estimation par télédétection de la fréquence d'apparition des fronts de brise de mer : Europe de l'ouest et Manche

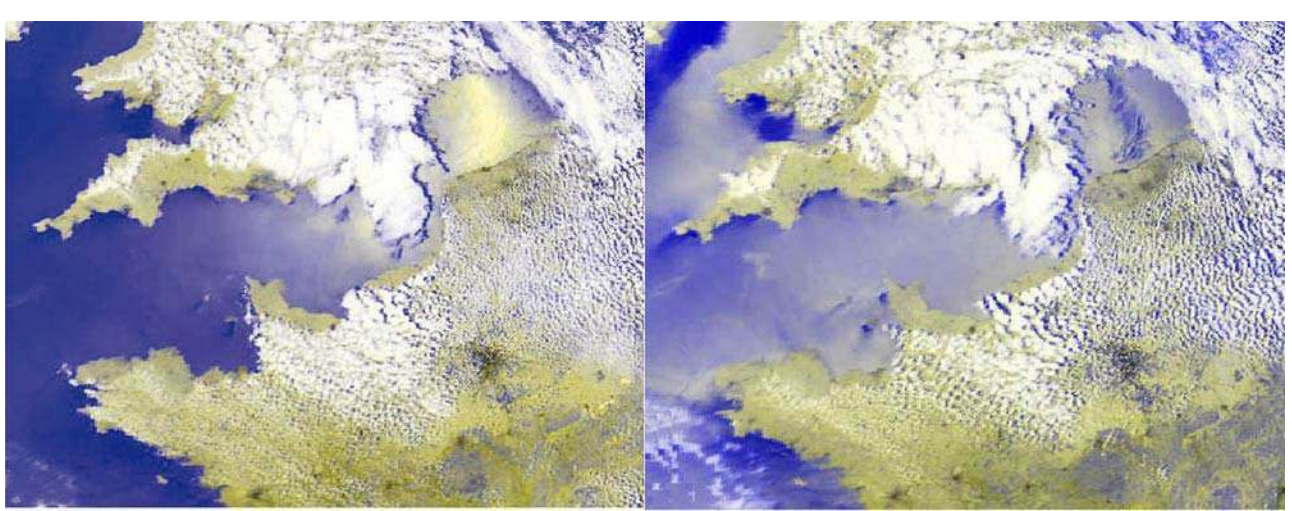

a) Images NOAA (compositions colorées) -14 / 26 juin 2000 à 14:00 UTC et à 15:00 UTC

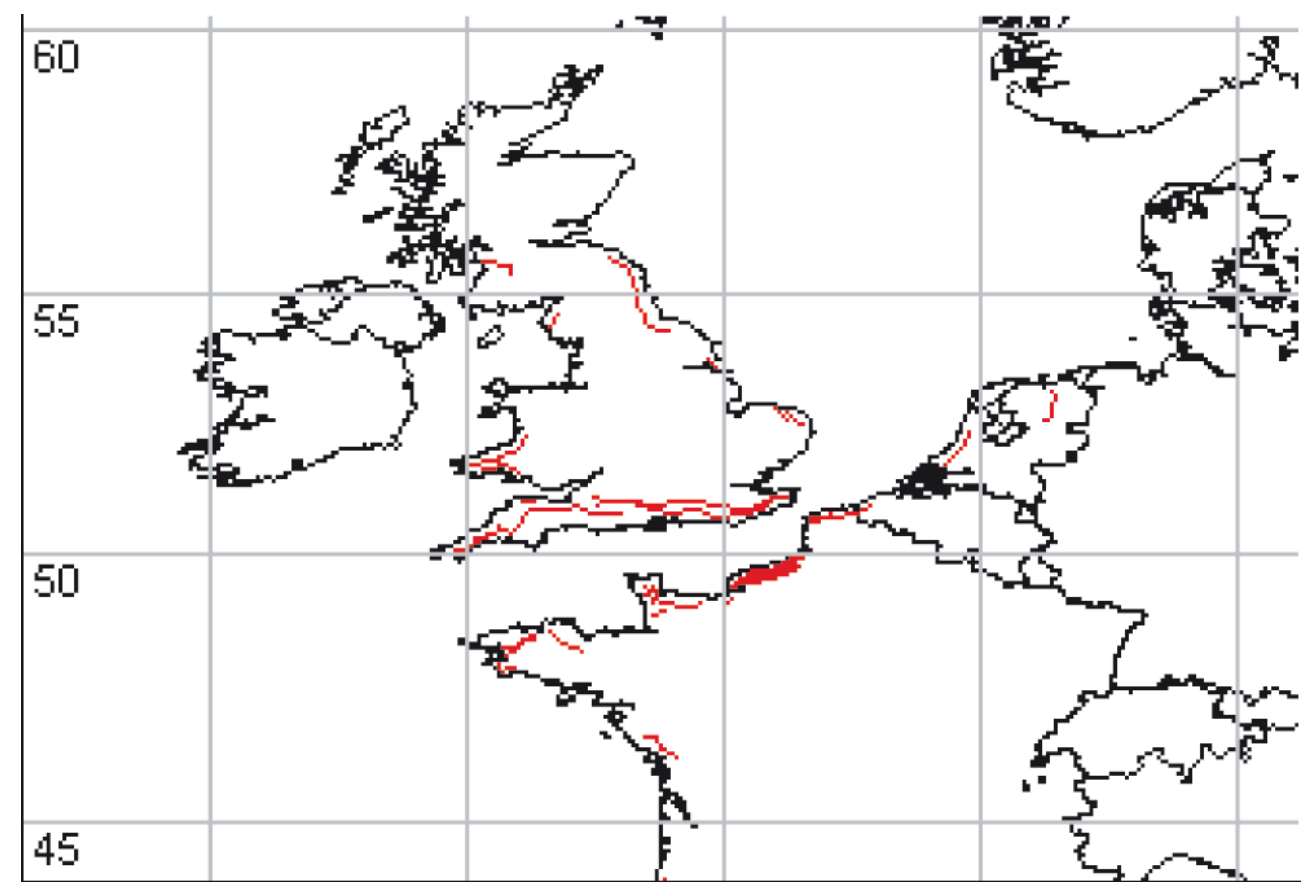

b) Fichiers vecteurs : carte de juin 2000 en Europe de l'Ouest. 


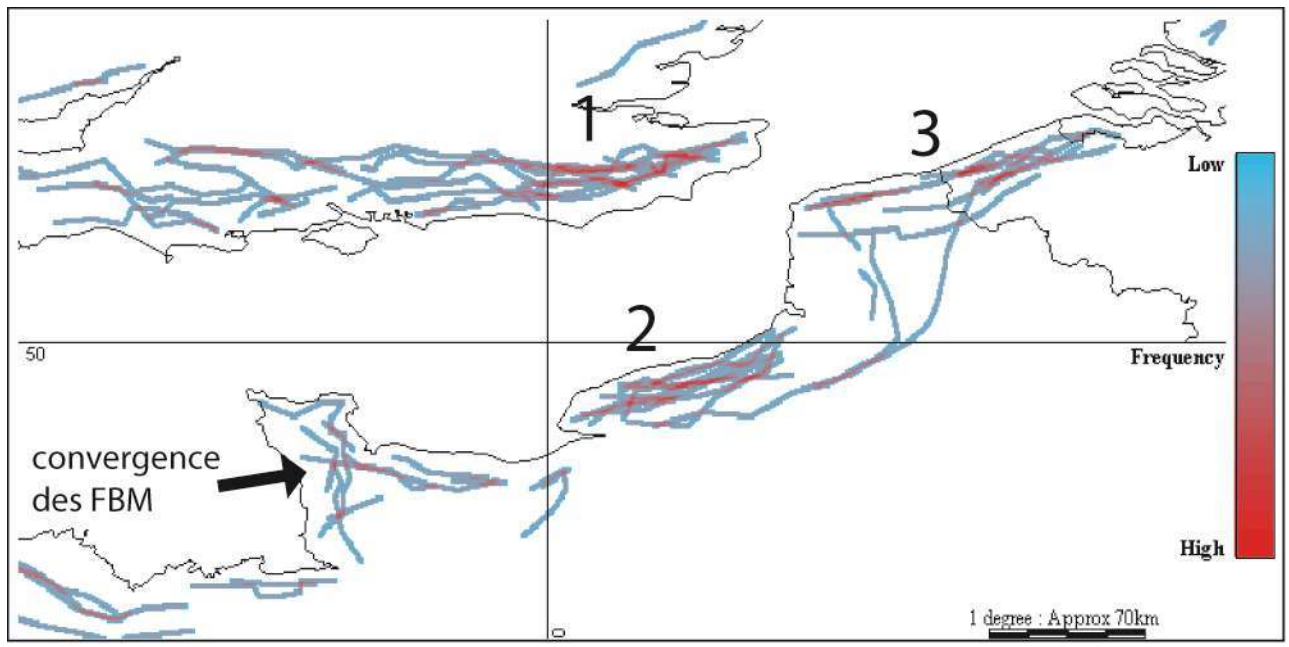

c) Fréquence d'observation des fronts de brise de mer autour de la Manche : synthèse pour la saison chaude (mai-septembre 2000).

Figure 6 : Estimation par télédétection de la fréquence d'apparition des fronts de brise de mer : Nord-Est du Brésil.

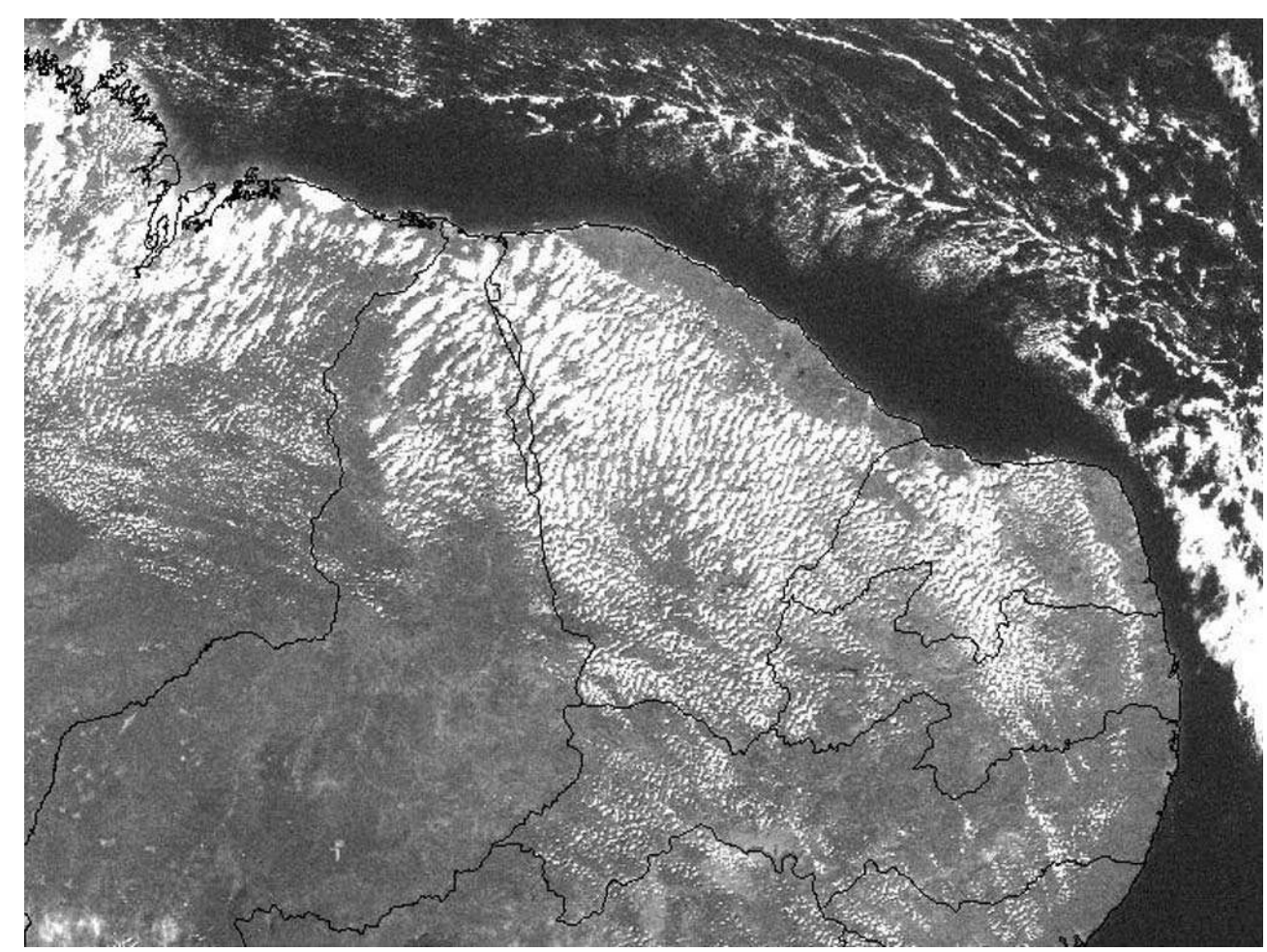

14 a) Image visible GOES-8 25 juin 1997 à 18:00 UTC. 


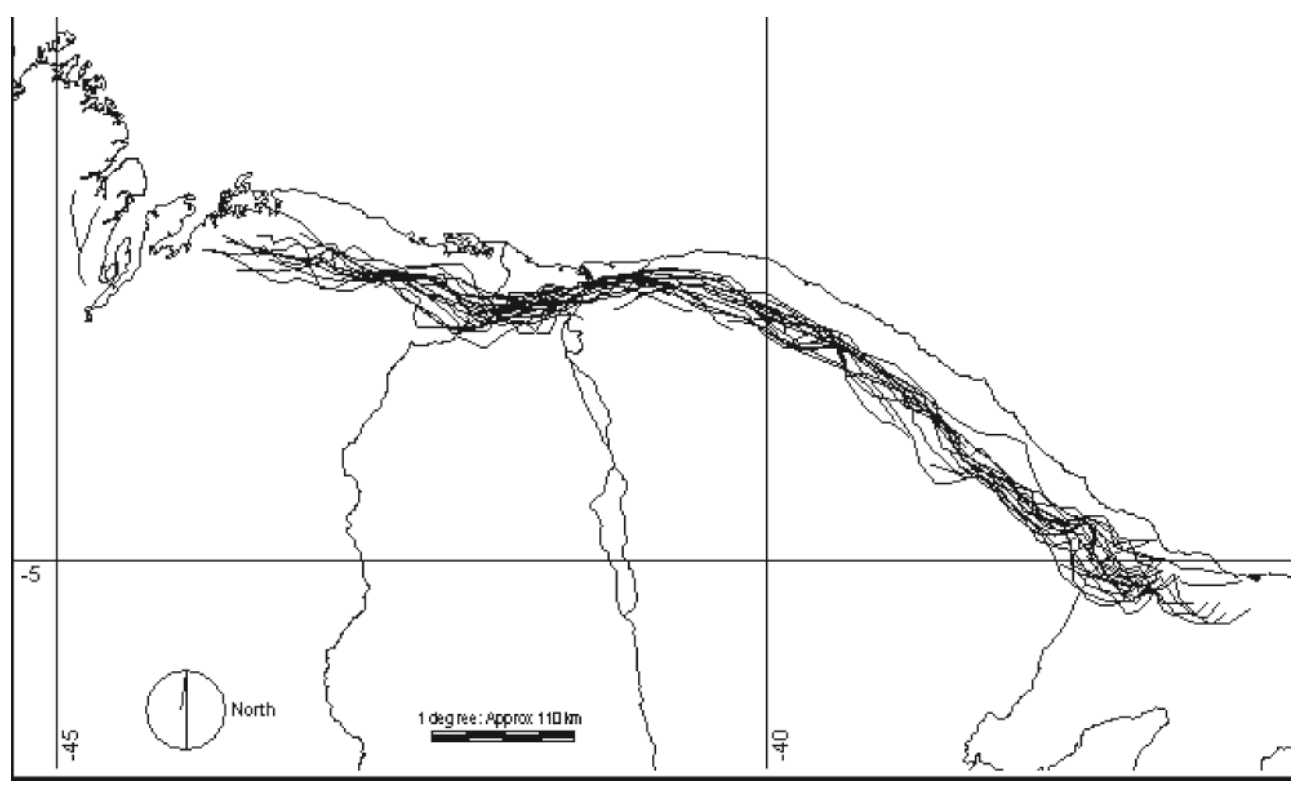

b) Fichiers vecteurs : carte de septembre 2000.

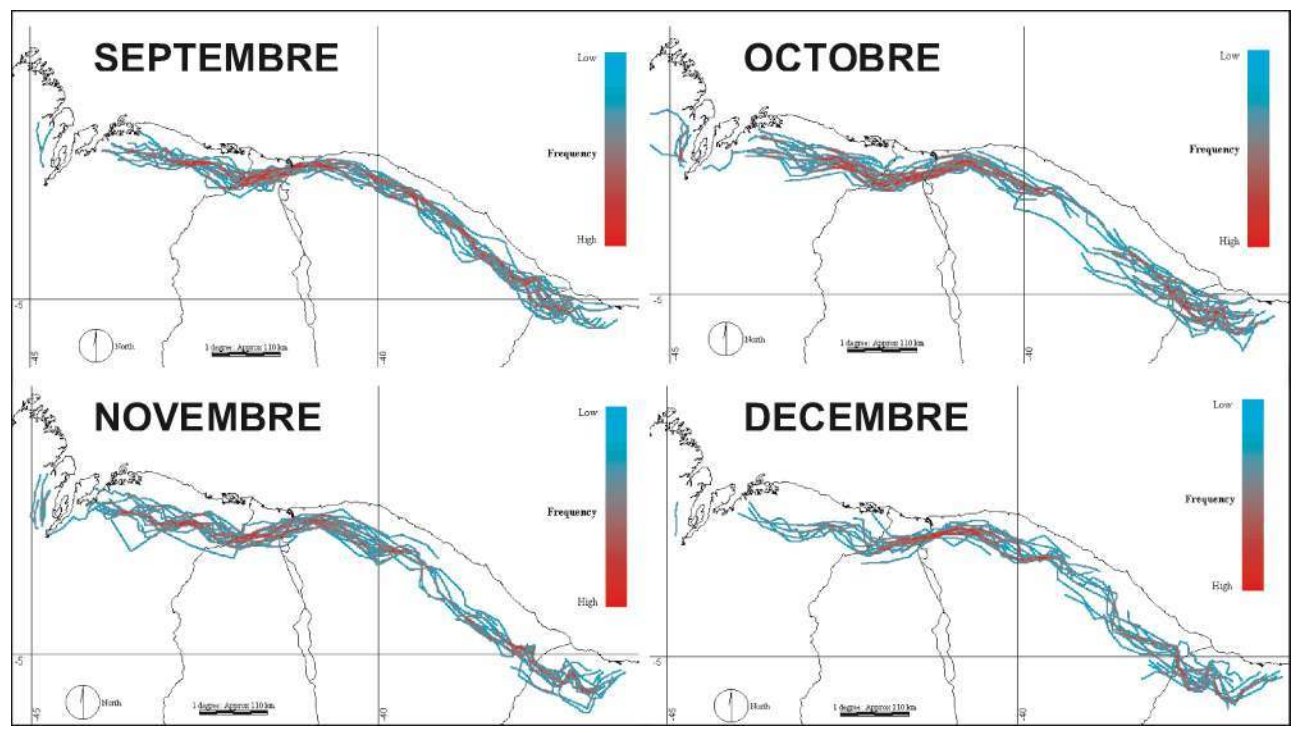

c) Fréquence d'observation des fronts de brise de mer sur le nord-est du Brésil pendant les mois de la saison sèche (septembre à décembre 2000)

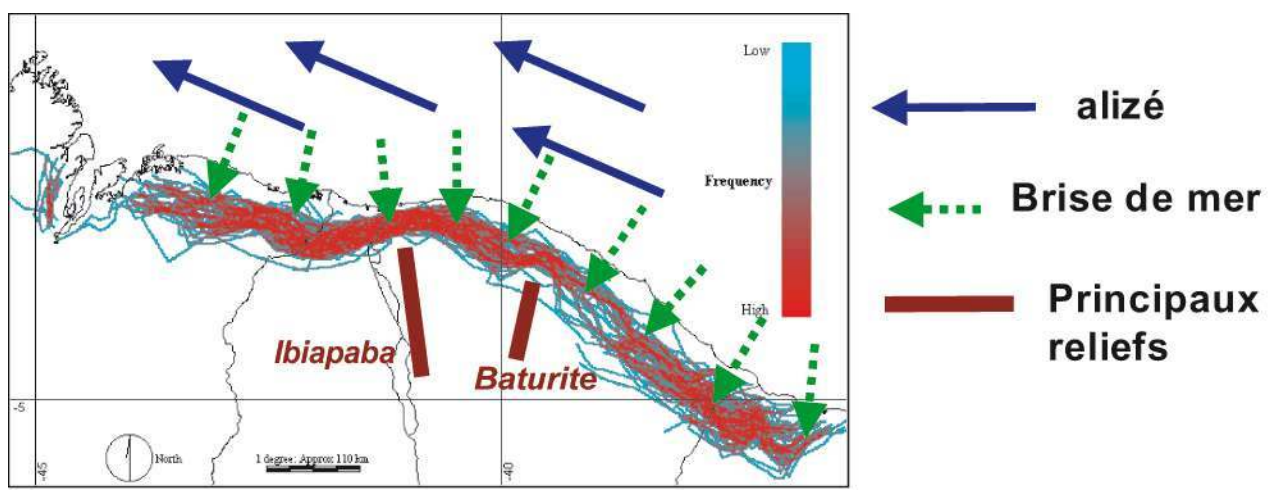

d) Fréquence d'observation des fronts de brise de mer sur le nord-est du Brésil pendant les mois de la saison sèche : synthèse saisonnière. 


\section{La distribution spatio-temporelle des fronts de brise}

\section{Autour de la Manche}

Les fronts de brise de mer ont été observés seulement dans le domaine tempéré océanique de l'Europe occidentale (partie nord de l'espace le plus large pris en compte). Aucun front de brise de mer n'a été identifié autour de la péninsule ibérique, en raison du ciel le plus souvent dégagé pendant la saison chaude: la méthode utilisée n'est pas adaptée aux climats de type méditerranéen. Malgré une distribution spatio-temporelle irrégulière durant la période étudiée, les fronts de brise de mer ont été observés le plus fréquemment autour de la Manche orientale à la fin du printemps et au début de l'été : sud-est de l'Angleterre (Sussex, Kent), Flandre belge et française et Haute-Normandie (Pays de Caux). La Manche et le Pas de Calais (incluant l'extrême sud de la mer du Nord) ont donc été étudiés de façon plus détaillée, entre 49 et $52^{\circ} \mathrm{N}$ (figure $5 \mathrm{c}$ ).

La topographie variée de plaines, bas plateaux et collines est associée à divers types de littoraux (dunes, falaises...) aux expositions variées. En Angleterre, les fronts de brise de mer ont le plus fréquemment été observés dans le Sussex et le Kent entre 20 et $40 \mathrm{~km}$ de la côte, en accord avec les observations de J.E. Simpson et al. (1977). Compte tenu des heures d'obervation (début de l'après-midi), les fronts de brise de mer franchissent habituellement les South Downs (alt. 200 à 300 m), mais pas les North Downs. Parmi les fréquences les plus élévées, la pénétration maximale a été enregistrée à $50 \mathrm{~km}$, dans le Wiltshire. Plus à l'est, la pénétration moindre mais très fréquente des fronts de brise suggère une convergence des brises de la Manche et de l'estuaire de la Tamise, comme l'a observé aussi J.E. Simpson (1994). En Normandie, les fronts de brise ont été le plus fréquemment observés sur le Pays de Caux, mais seulement entre 10 et $20 \mathrm{~km}$ dans l'intérieur. Les hautes falaises côtières ( 50 à $100 \mathrm{~m}$ ) exposées au nord ou au nord-ouest gênent la pénétration de la brise dans les terres (Escourrou, 1978). Ainsi, lorsque le flux dominant (d'échelle synoptique) et la brise étaient de même direction (vent de sud en Angleterre; de nord-ouest en Normandie), les fronts de brise pénétraient plus loin à l'intérieur de l'Angleterre que de la Normandie. Sur la péninsule du Cotentin (BasseNormandie), la distribution spatiale particulière des fronts de brise était fonction de l'articulation complexe des côtes. En Flandre, les fronts de brise ont été le plus fréquemment observés à seulement $10 \mathrm{~km}$ environ de la côte. Cependant, des fronts de brise ont été observés à $20 \mathrm{~km}$ de la côte, jusqu'aux collines des "Monts de Flandre" qui atteignent 150 à $180 \mathrm{~m}$. En Flandre belge, des fronts de brise ont été observés jusqu'à 30 $\mathrm{km}$ à l'intérieur. La plaine de Flandre maritime offre une topographie très plane, mais son littoral quasi rectiligne est exposé au nord et les flux dominants soufflent le plus fréquemment du sud-ouest (Leriche et al., 1998). En Artois et Boulonnais, la pénétration des fronts de brise de mer était trop variable pour mettre en évidence un seuil de fréquence élevée d'observations, car les brises y soufflent irrégulièrement de la mer du Nord et / ou de la Manche (Bigot et Planchon, 2003).

\section{Au nord-est du Brésil}

Sur la bordure nord-est du Brésil, la position des fronts de brise de mer montre un gradient spatial très resserré (figure 6c). Ce seuil est matérialisé par la forte densité des 
observations pour tous les mois. La topographie accidentée de la bordure exposée à l'est rend difficile l'identification des fronts de brise de mer, car ceux-ci sont souvent masqués par d'autres formations nuageuses liées aux effets orographiques. La bordure peu accidentée des Etats de Ceará, Piauí et Maranhão est plus favorable à la pénération des fronts de brise de mer dans l'intérieur des terres. A 18:00 UTC, la pénétration est d'autant plus importante que l'on s'avance dans la saison sèche : en septembre, les fronts de brise de mer ont été observés dans l'intérieur des terres à des distances de la côte comprises entre 40 et $80 \mathrm{~km}$, avec une fréquence maximale à environ $60 \mathrm{~km}$ de la côte. Le maximum de pénétration (60 à $100 \mathrm{~km}$ ) semblait atteint en novembre, mais seules des observations plus tardives en soirée permettraient de déterminer plus précisément quelle est la distance maximale de pénétration de la brise de mer. Dans les Guyanes et autour des bouches de l'Amazone, la distance de pénétration est probablement d'une centaine de kilomètres au moins, mais en pénétrant à l'intérieur, la nébulosité liée au front de brise se mêle à celle, de plus grande ampleur, liée à l'activité convective au-dessus des espaces forestiers (Snow, 1976 ; Cavalcanti, 1982 ; Molion, 1987).

La synthèse des fréquences pour toute la saison sèche (figure 6d) montre une large bande parallèle à la côte, située vers 40 à $100 \mathrm{~km}$ dans les terres et donnant ainsi une idée de l'extension du «climat maritime » sur l'espace étudié. Une étroite bordure de 20 à $40 \mathrm{~km}$ en moyenne a alors bénéficié fréquemment d'un ciel dégagé à l'heure de référence. En effet, quand le flux synoptique est de même sens que la brise de mer, le front de brise progresse rapidement vers l'intérieur dans la journée, le littoral bénéficiant alors de l'air maritime relativement stable et plus frais. Le tracé peu indenté de la côte facilite les observations de fronts de brise et permet de déterminer de quelle façon se module la pénétration des fronts en fonction de l'orientation de la côte. Les fronts de brise les mieux marqués et développés sont associés à un vent synoptique faible et de direction opposée à la brise de mer (Estoque, 1962 ; Arritt, 1993), mais un vent synoptique faible (moins de 6 ou $8 \mathrm{~m} \mathrm{~s}$-1 selon les auteurs: Borne et al., 1998) et de même sens que la brise de mer favorise la pénétration du front dans l'intérieur. Ainsi, les fronts de brise pénètrent plus loin dans les terres sur les bordures les mieux exposées aux alizés océaniques de sud-est (Ceará à l'est de $40^{\circ} \mathrm{W}$ et est du Maranhão) que sur les bordures exposées au nord ou NNW (Ceará à l'ouest de $40^{\circ} \mathrm{W}$ ), donc plus abritées que les précédentes. Dans cette région, les collines situées à proximité du littoral (Ibiapaba, Baturite) contribuent vraisemblablement à gêner la pénétration des fronts de brise. Ainsi, les fronts de brise sont le plus fréquemment observés, durant la saison sèche, à une distance du littoral variant peu d'un mois à l'autre. Les fronts de brise pénètrent moins loin dans l'intérieur (20 à $50 \mathrm{~km})$, leur progression est ralentie par les reliefs, même peu élevés, d'où un gradient spatial plus resserré qu'en arrière des côtes " au vent ». Les effets " au vent » et « sous le vent » se remarquent aussi de part et d'autre des baies de la région de São Luis (Maranhão) : les fronts de brise sont plus fréquemment observés en arrière de la rive occidentale, exposée aux alizés.

19 La fréquence d'apparition, la configuration et la distance de pénétration des fronts de brise de mer sont fortement influencés par les flux de plus large échelle, donc par les types de circulations d'échelle synoptique. Les circulations de brise de mer ne se développent qu'en présence de vents synoptiques faibles, ce qui rend les basses latitudes plus favorables au développement des brises que l'Europe du nord-ouest. De plus, le Brésil échappe aux cyclones tropicaux. Aux latitudes moyennes, la succession irrégulière de types de circulations et de situations météorologiques variés, même en été en domaine 
océanique, provoque une forte variabilité, d'un jour à l'autre et d'une année à l'autre, dans l'apparition des brises de mer et la capacité des fronts de brise à pénétrer dans l'intérieur des terres. Ainsi cette différence majeure entre les latitudes tempérées et tropicales s'est traduite par une meilleure pertinence des résultats des fréquences sur le nord-est du Brésil qu'autour de la Manche.

\section{Fronts de brise de mer, types de circulations et gradients thermiques terre-mer}

\section{Autour de la Manche : irrégularité des brises de mer liée aux faits circulatoires extra-tropicaux}

Une des conditions propices au développement d'une circulation de brise de mer nécessite en théorie un gradient thermique d'au moins $6^{\circ} \mathrm{C}$ aux hautes et moyennes latitudes (voir 1.1.). En réalité, cet écart thermique est un mauvais indicateur de l'intensité de la brise aux latitudes extratropicales, les conditions d'altitude et la circulation d'échelle synoptique étant également prédominantes (Savijärvi et Alestalo, 1988 ; Borne et al., 1998). Afin de relier les résultats de l'analyse par télédétection aux situations météorologiques d'échelle synoptique pour les mêmes journées d'observation, les Bulletins Hebdomadaires d'Etudes et de Renseignements de Météo-France (cartes synoptiques de surface et d'altitude / $500 \mathrm{hPa}$ à 12:00 UTC en Europe et sur l'océan Atlantique Nord) ont été dépouillés pour établir une classification. Les images enregistrées et analysées correspondaient principalement à des situations anticycloniques, en accord avec les observations de G.N. Sumner (1977) : les brises de mer se manifestent de préférence durant les situations anticycloniques et / ou caractérisées par l'intervention de masses d'air continental. Ces situations anticycloniques comprennent trois types principaux (figure 7) qui se partagent chacun les observations de fronts de brise de mer dans des proportions inégales.

Chaque situation a été définie en fonction de la position des hautes pressions de surface. La situation à anticyclone nord-européen a été la plus favorable aux observations de fronts de brise de mer en Europe de l'ouest (54\% du total des observations), en juin notamment. Cette situation est surtout fréquente au printemps: les eaux de surface encore fraîches de la Manche et de la mer du Nord favorisent une anticyclogenèse sur les Iles Britanniques, voire des Iles Britanniques aux pays Baltes (Kendrew 1961; Moron 1990). Ainsi, la fréquence la plus élevée d'observations de fronts de brise a été relevée autour de la Manche et de la mer du Nord (respectivement 14 et 11 observations : Damato et al., 2003). Les hautes pressions de surface nord-européennes protègent directement ces deux espaces maritimes des perturbations atlantiques. Le 16.06.2000 (Fig. 7a), un anticyclone centré sur la mer du Nord dirigeait des vents de nord-ouest au-dessus de la mer du nord et d'est au-dessus de la Manche. Des fronts de brise de mer ont ainsi été observés en Hollande et en Flandre (brise de mer de même direction que le vent dominant), mais aussi en Angleterre du sud-est et en Normandie (pays de Caux) 
Figure 7 : Images visibles et situations météorologiques pour trois journées sélectionnées (avec position des fronts de brise de mer observés en lignes noires épaisses).
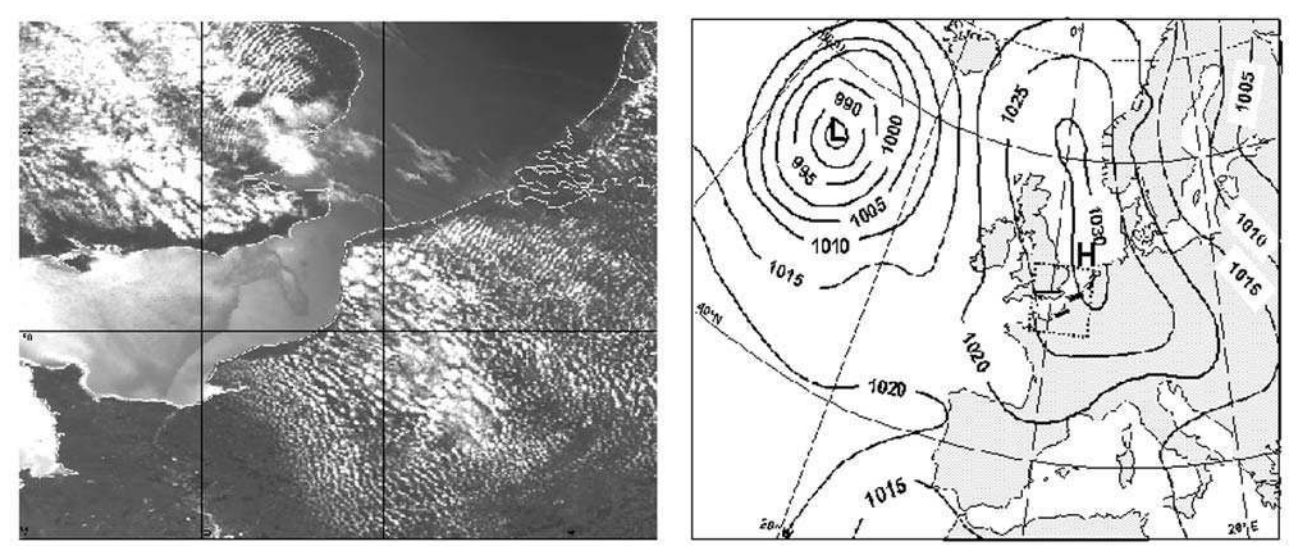

a) 16 juin 2000 : Anticyclone nord-européen
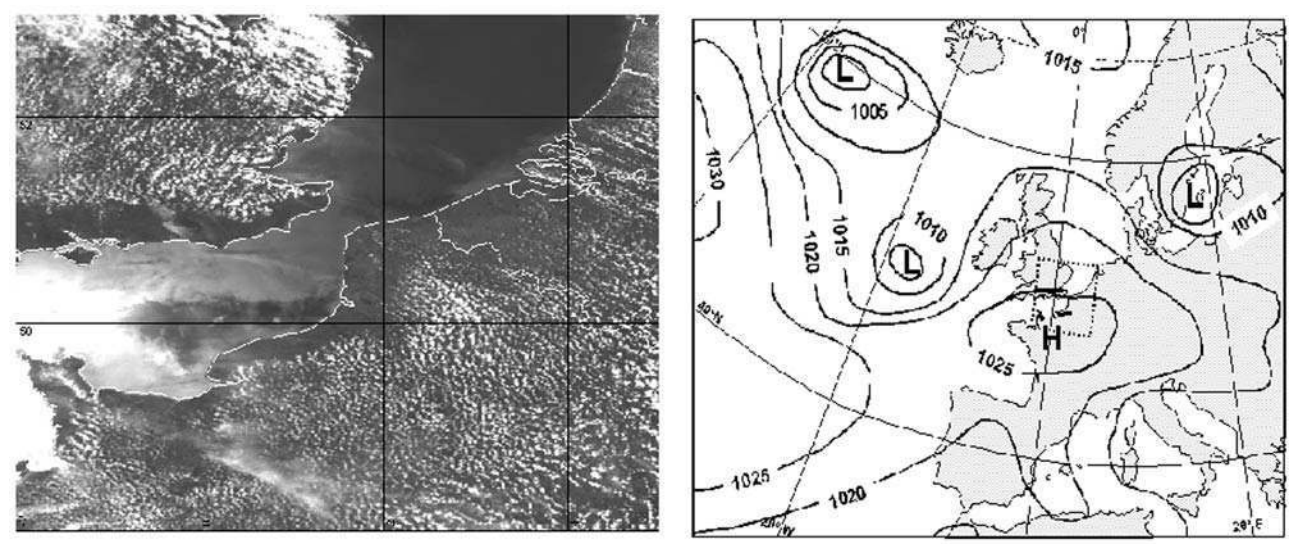

b) 7 juin 2000 : Anticyclone sur l'Europe moyenne
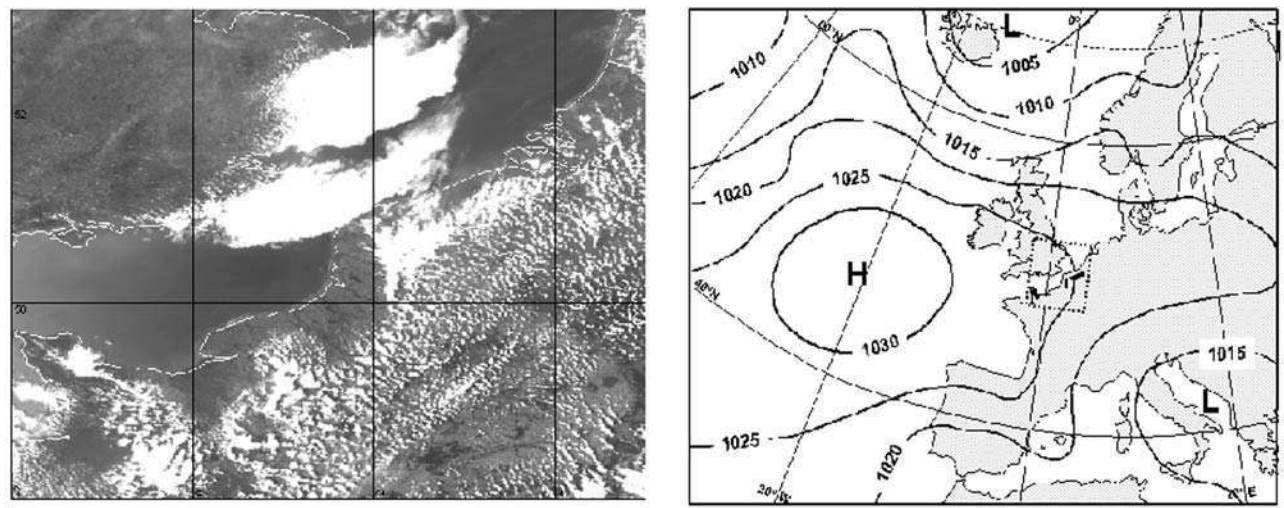

c) 5 août 2000 : Anticyclone atlantique

Les situations à hautes pressions de surface sur l'Europe moyenne (sur la France, avec extension variable dans l'espace vers l'Europe centrale) representaient $31 \%$ des observations de fronts de brise de mer sur l'Europe de l'ouest. La plus grande partie de l'Europe de l'ouest était abritée des perturbations atlantiques qui touchaient alors les Iles Britanniques et l'Europe du Nord: seule une observation de front de brise a été détectée dans l'ouest et le nord des Iles Britanniques. Les vents dominants d'ouest ou de sud-ouest gênaient ou empêchaient le développement des circulations de brise de mer. Le 
07.06.2000 (Fig. 7b) un anticyclone sur l'ouest de la France, avec extension sur le centre et le nord de l'Europe, rejetait les trajectoires des sytèmes perturbés sur l'Europe du nord, ce qui a permis d'observer des fronts de brise sur les deux bordures de la Manche (Angleterre, pays de Caux et Cotentin). Les côtes de ces régions étaient exposées à des flux d'ouest ou de sud-ouest : c'est ainsi, dans le sud de l'Angleterre, que les fronts de brise ont pénétré le plus loin à l'intérieur.

Les situations à hautes pressions de surface sur l'océan Atlantique Nord étaient les moins favorables à l'observation des fronts de brise de mer sur l'Europe de l'Ouest (15\% du total des observations), puisque celle-ci était moins protégée des perturbations atlantiques. Deux types de situations pouvaient cependant être différenciées :

1. Position méridionale de l'anticyclone atlantique : un flux de sud-ouest perturbé circule sur l'Europe du nord-ouest.

2. Position septentrionale de l'anticyclone atlantique: un flux de nord-ouest circule sur l'Europe de l'ouest, davantage abritée des systèmes perturbés. Cette situation était donc plus favorable que la précédente aux observations de fronts de brise de mer autour de la Manche.

Le 05.08.2000 (fig. 7c), le centre de hautes pressions de surface (> $1030 \mathrm{hPa}$ ) était situé audessus de l'océan Atlantique entre 40 et $50^{\circ} \mathrm{N}$, dirigeant les systèmes perturbés sur les Iles Britanniques et la Scandinavie. Les vents dominants d'WNW sur la Manche et la mer du Nord ont permis le développement d'une large bordure de ciel dégagé de la Normandie à la Flandre, notamment sur le pays de Caux et l'Artois, mais seules les lignes de nuages situées au-dessus de la Flandre belge, de l'Artois et de la Normandie occidentale pouvaient être définies comme des fronts de brise de mer.

\section{Alizé et brise de mer au nord-est du Brésil : une interaction marquée par la régularité}

La démarche fondée sur la variabilité des types de situations météorologiques et des circulations d'échelle synoptique, efficace en Europe, ne peut pas s'appliquer aux très basses latitudes telles que dans le nord-est du Brésil compte tenu de faits circulatoires plus réguliers qu'aux latitudes extratropicales (climat rythmé par le balancement latitudinal saisonnier de la ZCIT). D'autres aspects faisant intervenir l'évolution saisonnière des éléments du climat peuvent être retenus, en intégrant le rôle joué par les états de surface. Le réchauffement différentiel entre la surface de l'océan et la bordure continentale crée un gradient thermique plus ou moins favorable à la pénétration des fronts de brise de mer dans l'intérieur des terres. La différence entre la moyenne des températures maximales de surface sur la bordure du continent et la moyenne des températures maximales sur l'océan, déterminée à partir des données infrarouge GOES-8, a permis d'estimer le gradient thermique terre-mer (Tableau 1).

Tableau 1 : Evolution mensuelle du gradient thermique de surface terre-mer (données infrarouge GOES-8).

\begin{tabular}{|l|l|l|l|l|l|l|}
\hline & Sep & Oct & Nov & Déc & SP & SS \\
\hline CTM & 5,3 & 6,7 & 6,2 & 7,1 & 4,5 & 6,3 \\
\hline $\mathrm{F} \%>4^{\circ} \mathrm{C}$ & 94,1 & 89,3 & 90,9 & 100 & 64,1 & 93,5 \\
\hline
\end{tabular}




\begin{tabular}{|l|l|l|l|l|l|l|}
\hline СтмСS & 5,3 & 6,7 & 6,4 & 7,4 & 5.0 & 6,4 \\
\hline \hline СтмСо & - & 6,2 & 3,9 & 5,8 & 4,1 & 5,3 \\
\hline
\end{tabular}

CTM : contraste thermique terre-mer. $\mathrm{F} \%>4^{\circ} \mathrm{C}$ :fréquence mensuelle et saisonnière (en \%) des contrastes thermiques supérieures au seuil de $4^{\circ} \mathrm{C}$. CS : côte septentrionale. CO : côte orientale. SP : saison pluvieuse. SS : saison sèche.

Un gradient thermique d'au moins $4^{\circ} \mathrm{C}$, entre la surface du continent et celle de la mer, est théoriquement nécessaire au développement des circulations de brise de mer aux latitudes intertropicales (voir 1.1.). En 2000, au pas de temps mensuel, les contrastes thermiques terre-mer étaient le plus souvent supérieurs à $4^{\circ} \mathrm{C}$. Cependant, l'ampleur de ce contraste thermique présentait des variations saisonnières imposées par l'alternance saison pluvieuse/saison sèche. 93,5\% des observations étaient associées à des gradients thermiques supérieurs à $4^{\circ} \mathrm{C}$ en saison sèche, contre $64,1 \%$ en saison pluvieuse. La brise de mer a pu théoriquement se produire quasiment tous les jours durant la saison sèche, ce qui confirme les observations réalisées dans le visible. Les contrastes thermiques étaient compris entre 6 et $7^{\circ} \mathrm{C}$ en moyenne, mais ils s'amplifiaient de septembre $\left(5-6^{\circ} \mathrm{C}\right)$ à décembre $\left(7-8^{\circ} \mathrm{C}\right)$. Cette évolution est liée à l'augmentation de la température de surface sur le continent en relation avec l'efficacité de la radiation solaire en cette saison, associée à une nébulosité faible. L'accentuation du gradient thermique de surface terremer au cours de la saison sèche permet donc la mise en place de conditions thermiques favorables au développement de circulations de brises de mer puissantes capables de pénétrer d'autant plus loin dans l'intérieur, les alizés apportant l'humidité atmosphérique nécessaire à la formation de nuages au niveau du front de brise. De plus, il semble qu'à partir du seuil de $4^{\circ} \mathrm{C}$, un écart de $1^{\circ} \mathrm{C}$ de plus équivaut en moyenne à $10 \mathrm{~km}$ supplémentaires parcourus par le front de brise (Damato, 2002).

Les brises de mer acquièrent ainsi une grande régularité et une puissance particulière sous les climats intertropicaux à saison sèche. A l'est des continents, la pénétration dans l'intérieur est favorisée par les alizés océaniques, de même direction que la brise. Les circulations de brise s'inscrivent dans la circulation des alizés, dont elles peuvent régulièrement infléchir la direction dans les basses couches mais en renforcent la vitesse dans l'après-midi. La direction du vent est aussi nuancée en fonction de la saison, par exemple en Tanzanie et au Kenya (Sumner, 1984 ; Camberlin et Planchon, 1997). Dans le nord-est du Brésil, le rythme diurne de la direction du vent en surface traduit bien la combinaison de l'alizé océanique et de la brise de mer, la déviation du vent de surface étant fonction également de l'orientation de la côte (Figure 8). 
Figure 8 : Fréquence de la direction du vent pour trois stations du Nord-Est du Brésil en septembre 2001 (données Météo-France).

FORTALEZA

(Latitude $3.78 \mathrm{~N}$; Longitude $38.53 \mathrm{~W}$; Altitude $25 \mathrm{~m}$ )
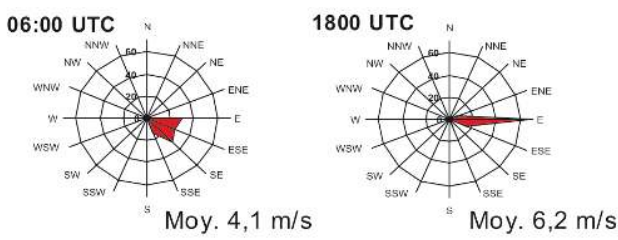

NATAL

(Latitude $5.92 \mathrm{~N}$; Longitude $35.25 \mathrm{~W}$; Altitude $49 \mathrm{~m}$ )
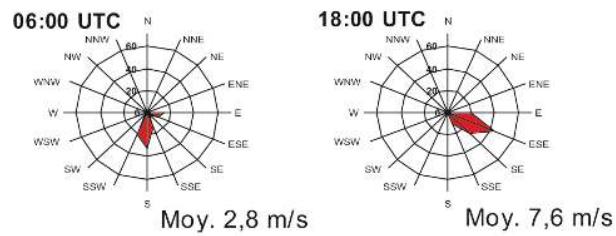

RECIFE

(Latitude $8.05 \mathrm{~N}$; Longitude $34.92 \mathrm{~W}$; Altitude $11 \mathrm{~m}$ )
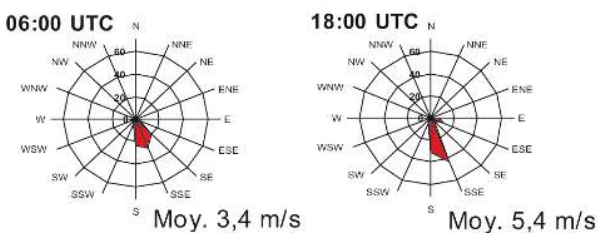

Ainsi, en septembre 2001, le vent s'orientait de préférence, à Fortaleza (côte exposée au NE) : du SE à $06: 00$ UTC à l'E à 18:00 UTC; à Natal (côte exposée à l'ENE) : du S ou SSW (06:00 UTC) à l'ESE (18:00 UTC). A Recife (côte exposée à l'ESE), la direction restait essentiellement orientée du SSE à S, avec cependant un renforcement de la fréquence de ces deux directions (15.4\% de calmes à 06:00 UTC), et un renforcement de la fréquence des directions $\mathrm{E}$ et notamment ESE (11.5 \%). Ces changements diurnes traduisent l'alternance brise de terre (à 06:00 UTC) / brise de mer (à 18:00 UTC), dont les directions subissent à la fois l'influence de l'alizé et de l'orientation de la côte.L'effet des circulations de brises sur la direction du vent est aussi bien marqué à Natal qu'à Fortaleza. A Recife, la régularité de l'alternance journalière de brises a déjà été mise en évidence (Vulquin, 1979). En septembre 2001, la vitesse moyenne du vent en surface se renforçait sur les trois stations (Fortaleza : 4.1 à $6.2 \mathrm{~m} \mathrm{~s}-1$, Natal : 2.8 à $7.6 \mathrm{~m} \mathrm{~s}$-1 et Recife : 3.4 à $5.4 \mathrm{~m} \mathrm{~s}-1$, entre 06:00 et 18:00 UTC), ce qui traduisait l'intervention répétée des brises de mer se combinant à l'alizé. Ce n'est donc pas l'absence de circulations de brise de mer qui est en cause dans l'absence d'observations de fronts de brise sur la bordure exposée à l'est, mais essentiellement un problème du au relief. Les massifs montagneux introduisent un niveau de complexité supplémentaire dans l'interprétation des images, par la nébulosité qu'ils favorisent fréquemment sur la bordure du continent. La plaine côtière est assez étroite (50 à $100 \mathrm{~km}$ ) et la nébulosité éventuelle liée aux fronts de brise se mêle donc rapidement vers l'intérieur à celle liée à la convection et / ou aux ascendances orographiques liées aux massifs montagneux.

La distance de pénétration des fronts de brise varie en fonction de l'exposition de la côte aux vents dominants, en Europe comme au Brésil. Cependant, la succession de situations météorologiques variées impose des configurations diverses d'un mois à l'autre en Europe 
de l'Ouest, tandis de la régularité des alizés fait ressortir une plus grande permanence, dans l'espace et dans le temps, de la localisation des fronts de brise au nord-est du Brésil. Grâce à la pénétration de la brise de mer à plusieurs dizaines de kilomètres dans l'intérieur des terres, le temps ensoleillé, à ciel dégagé, est habituel dans l'après-midi sur la bordure de l'État du Ceará.

\section{Conclusion}

Une méthode d'identification des fronts de brises par télédétection a été appliquée à l'Europe de l'Ouest (plus spécialement autour de la Manche) et au nord-est du Brésil (plus spécialement sur la bordure des Etats de Ceará, Piauí et Maranhão), afin d'en calculer la fréquence d'apparition et la distance préférentielle de pénétration dans les terres aux heures chaudes de la journée (début de l'après-midi en Europe ; 18:00 UTC au Brésil). Alors que la succession des types de circulation et de situations météorologiques variées, même en été, rend irrégulière l'apparition et l'intensité des brises de mer autour de la Manche, la régularité de la circulation des alizés et la charge hygrométrique élevée de l'air tropical océanique est favorable, même durant les mois les moins arrosés ou saison «sèche» (peu de pluie, mais un air humide sous l'influence des alizés océaniques) au développement de formations nuageuses convectives (de type cumuliforme) associées aux fronts de brise de mer. La structure particulière et nette de ces formations nuageuses a permis d'établir des cartes de fréquences durant la saison chaude (mai à septembre) autour de la Manche et surtout durant la saison sèche au nord-est du Brésil (septembre à décembre), mois par mois et tous mois confondus.

31 Les résultats confirment d'une part (au Brésil) que les fronts de brise pénètrent d'autant plus loin dans les terres que l'on s'avance au cœur de la saison sèche (renforcement du gradient thermique terre-mer), et, d'autre part (au Brésil et en Europe), que la distance de pénétration des fronts de brise varie en fonction de l'exposition de la côte aux vents dominants. Au nord-est du Brésil, même sur les bordures les moins bien exposées, les cartes de fréquences font ressortir une permanence dans l'espace et dans le temps (mois par mois) de la position des fronts de brise à 18:00 UTC. Autour de la Manche, une classification des types de situations météorologiques reposant sur la configuration du champ de pression en surface a permis de hiérarchiser les principaux types de situations météorologiques favorables aux observations de fronts de brise, en fonction de la fréquence d'observation de ceux-ci. La validation des résultats obtenus par l'analyse des données de télédétection nécessite une confrontation de ces résultats avec des données météorologiques : en climat tropical humide par exemple, l'activité convective suscitée par l'arrivée du front de brise de mer sur le continent est suffisante pour provoquer des averses et mettre en place, du fait de la régularité des circulations de brises, des rythmes pluviométriques diurnes spécifiques aux climats tropicaux côtiers et maritimes. Des données pluviométriques horaires permettraient de le vérifier sur le nord-est du Brésil, et d'en étudier les nuances en fonction des expositions et des saisons. Dans le nord de la France, une technique algébrique, la Singular Value Decomposition (SVD) a permis très récemment d'identifier des journées à brise de mer sur la région Nord-Pasde-Calais à partir de données radiométriques NOAA-AVHRR et des données de température de surface des stations disponibles (Bigot et Planchon, 2003). 


\section{BIBLIOGRAPHIE}

ABBS, D.J. et PHYSICK, W.L. (1992) « Sea breeze observations and modelling: a review. » Australian Meteorological Magazine, 41, pp.7-19.

ARRITT, R.W. (1993) « Effects of the large-scale flow on characteristic features of the sea breeze.» Journal of Applied Meteorology, 32, pp. 116-125.

BIGOT, S. et PLANCHON, O. (2003) «Identification and characterisation of sea breeze days in Northern France using singular value decomposition. » International Journal of Climatology, 23(11), 1387-1405.

BORNE, K., CHEN, D. et NUNEZ, M. (1998) « A method for finding sea breeze days under stable synoptic conditions and its application to the swedish west coast. » International Journal of Climatology, 18 (8), 901-914.

BONNARDOT, V., PLANCHON, O., CAREY, V.A. et CAUTENET, S. (2002) » Diurnal wind, relative humidity and temperature variation in the Stellenbosch-Groot Drakenstein winegrowing area. » South African Journal of Enology and Viticulture, 23 (2), 62-71.

CAMBERLIN, P. et PLANCHON O. (1997) «Coastal precipitation regimes in the equatorial latitudes, the case of Kenya. » Geografiska Annaler, 79 A (1-2), pp. 109-119.

CAUTENET S. (1988) Interaction d'une circulation méso-échelle de brise de mer avec un cisaillement de vent synoptique. Application au golfe de Guinée. Thèse de Doctorat, Université Blaise Pascal, ClermontFerrand, $204 \mathrm{p}$.

CAUTENET, S. , ROSSET, R. (1989) « Numerical simulation of sea breezes with vertical wind shear during dry season at Cape of Three Points », West Africa. Monthly Weather Review, 117, 329-339.

CAVALCANTI, I.F.A. (1982) Um estudo sobre interações entre sistemas de circulação de escala sinótica e circulações locais. MSc thesis, São José dos Campos: INPE-2494-TDL/097.

DAMATO, F., PLANCHON, O. et DUBREUIL, V. (2003a) « A remote sensing study of the inland penetration of sea breeze fronts from the English Channel », Weather, 58 (6), 219-225.

DAmato, F., Planchon, O., DUbreuil, V. et GOUery, P. (2003b) : « Etude par télédétection de la localisation des fronts de brise de mer dans le Nord-Est du Brésil. » Anais XI SBSR, Belo Horizonte (Brésil), INPE, 1165-1172 (sur CD).

DAMATO, F. (2002) Contribution à l'étude de la pénétration des fronts de brise de mer au Nordeste (Brésil). Analyse d'après les données infrarouge du satellite GOES-8. Mémoire de D.E.A., Université de Rennes-2, $70 \mathrm{p}$.

DECAUX, S. (2002) Le climat de la côte d'Emeraude (Bretagne / Manche). Analyse statistique et caractéristiques locales des circulations de brises et de leurs effets. Mémoire de Maîtrise, Université de Rennes-2, 86 p.

Escourrou, G. (1978) Climats et types de temps en Normandie. Doctoral Thesis, Champion, Paris, 1081 $+525 \mathrm{pp}$.

ESTOQUE, M.A. (1962) « The sea breeze as a function of the prevailing synoptic situation.Journal of Atmospheric Science », 19, 244-250. 
JANOUEIX-YACONO, D. (1995) « Rapports entre brise de mer ou de lac, structure de la couche limite planétaire et pollution atmosphérique sur les plaines littorales urbanisées. » Climat, Pollution atmosphérique, Santé (Hommage à Gisèle Escourrou), 177-201.

KENDREW, W.G. (1961) The climates of the continents. Oxford at the Clarendon Press, Oxford University Press, London, 608 pp.

LERICHE, P., KERGOMARD, C. et CHAPELET, P. (1999) «Etude des brises de mer sur le littoral français de la mer du Nord. Publications de l' Association Internationale de Climatologie, 11, 107-114.

MAYEnçON, R. (1992) Météorologie marine. Ed. Maritimes \& d'Outre-Mer, 336 p.

MOLION, L.C.B. (1987) « On the dynamic climatology of the Amazon basin and associated rainproducing mechanisms. » In : The geophysiology of Amazonia, Ed. Dickinson R.E., Wiley, 391-407. MORON, V. (1990) Contribution à l'étude de la variabilité climatique mensuelle en Europe. Mémoire de D.E.A., Université de Bourgogne, Dijon.

MOUNIER, J. (1982) «Brouillards d'été sur les côtes septentrionales de la Bretagne. Norois, 116, 547-559.

MOUNIER, J. et GOUÉRY, P. (1992) » Température de la mer autour du Massif Armoricain selon les données des satellites NOAA-AVHRR. » Norois, 155, 305-315.

NIMER, E. (1989) Climatologia do Brasil. IBGE, Rio de Janeiro, 421 p.

O'HARE, G. et SWEENEY, J. (1994) The atmospheric system. Oliver \& Boyd, Longman, Harlow (UK), 208 p.

OKOOLA, R.E.A. (1978) « Spatial distribution of precipitation in the Mombasa area of Kenya, Kenya Meteorological Department, IMTR Research Report, 1/78.

PIELKE, R.A. (1974) « A three-dimensional numerical model of sea breezes over South Florida. Monthly Weather Review, 102, 115-139.

PLANCHON, O. (1997) Les climats maritimes dans le Monde. Presses Universitaires du Septentrion, Vitteneuve d'Ascq, $233 \mathrm{p}$.

PLANCHON, O. (2000) « A study of the coastal climates in France using temperature and precipitation data (1961-1990). Meteorological Applications, 7, 217-228.

PLANCHON, O., BONNARDOT, V. et CAUTENET, S. (2000) « Simulation de brise de mer (résolution à 5 $\mathrm{km}$ ) : exemple de la journée du 4 février 2000 dans la Province Occidentale du Cap. » Publications de l'Association Internationale de Climatologie, Nice, 13, 482-489.

RATISBONA, L. R. « The climate of Brazil. » In: World Survey of Climatology, Amsterdam: Elsevier, 1976, 12 (Chap. 5), 219-293.

SAVIJÄRVI, H. et ALESTALO M. (1988) « The sea breeze over a lake or gulf as the function of the prevailing flow. » Beiträge zur Physik der Atmosphäre, 61 (2), 98-104.

SIMPSON, J.E. (1994) Sea breeze and local winds. Cambridge University Press, 234 p.

SIMPSON, J.E., MANSFIELD, D.A. and MILFORD, J.R. (1977) « Inland penetration of sea breeze fronts. » Quarterly Journal of the Royal Meteorological Society, 103, 47-76.

SMITH, K. (1976) « The climates of coasts and inland water bodies. » In : The climate of the British Isles. I.J. Chandler, S. Gregory, Longman, 248-263. 
SNOW, J.W. (1976) « The climate of Northern South America. » In: World Survey of Climatology, Amsterdam: Elsevier, 12 (Chap. 6), 295-403.

SUMNER, G.N. (1977) « Sea breeze occurrence in hilly terrain. Weather, 32, pp.200-208.

Vulquin, A. (1979) Comparaison entre le climat de Buenos Aires et les climats de la façade orientale du Brésil. Thèse de Doctorat d'État - Université de Bourgogne, Dijon, 2, 552 p.

YAN, H.; ANTHES, R.A. « The effect of latitude on the sea breeze. » Monthly Weather Review, 115 (5), 936-956, 1987.

\section{RÉSUMÉS}

Les circulations de brise de mer favorisent l'apparition de types de temps relativement frais et humides mais le plus souvent ensoleillés sur les espaces côtiers par rapport aux régions intérieures. Une méthode d'identification des fronts de brise par télédétection a été appliquée à l'Europe de l'Ouest et au nord-est du Brésil, afin d'en calculer la fréquence d'apparition et la distance préférentielle de pénétration dans les terres aux heures chaudes de la journée. Le suivi des fronts de brise a été effectué durant la saison chaude en Europe occidentale (mai à septembre 2000) et la moins arrosée dans le Nord-Est du Brésil (septembre à décembre 2000). La distance de pénétration des fronts de brise varie en fonction de l'exposition de la côte aux vents dominants, en Europe comme au Brésil. Cependant, la succession de situations météorologiques variées impose des configurations diverses d'un mois à l'autre en Europe de l'Ouest, tandis de la régularité des alizés fait ressortir une plus grande permanence, dans l'espace et dans le temps, de la localisation des fronts de brise au nord-est du Brésil.

The sea breeze circulations involve cool and damp but sunny weather types over coastal fringes compared to inland areas. A method of identification of the sea breeze fronts was performed and applied to Western Europe and North-eastern Brazil, in order to calculate the occurrence of sea breeze fronts during the warmest period of the day and to calculate the most frequent distance of inland penetration. The sea breeze front monitoring was carried out during the warm season in Western Europe (May-September 2000) and during the less rainy season in North-eastern Brazil (September-December 2000). In Europe and Brazil, the inland penetration of sea breeze fronts varied according to the exposure of the coast to the prevailing wind. The succession of varied meteorological situations involved varied spatial organisation of the sea breeze fronts in Western Europe, while the more regular trade winds circulation allowed a less variable space and time organisation of the sea breeze fronts in the northeast of Brazil.

\section{INDEX}

Index géographique : Brésil, Europe de l'Ouest

Keywords : remote sensing, sea breeze

Mots-clés : brise de mer, télédétection

\section{AUTEURS}

\section{OLIVIER PLANCHON}

COSTEL (LETG-UMR 6554 CNRS) - Université Rennes 2,olivier.planchon@uhb.fr 
VINCENT DUBREUIL

COSTEL (LETG-UMR 6554 CNRS) - Université Rennes 2, vincent.dubreuil@uhb.fr

FRÉDÉRIC DAMATO

COSTEL (LETG-UMR 6554 CNRS) - Université Rennes 2

PASCAL GOUÉRY

COSTEL (LETG-UMR 6554 CNRS) - Université Rennes 2

SÉBASTIEN DECAUX

COSTEL (LETG-UMR 6554 CNRS) - Université Rennes 2 